\title{
II ruolo della galectina-3 nella patologia cardio-renale
}

\author{
Luca Di Lullo ${ }^{1}$, Rodolfo Rivera ${ }^{2}$, Fulvio Floccari ${ }^{3}$, Vincenzo Barbera' ${ }^{1}$, Antonio Bellasi ${ }^{4}$, Antonio De Pascalis ${ }^{5}$, Domenico Russo ${ }^{6}$, \\ Luigi Russo ${ }^{6}$, Francesca Santoboni ${ }^{1}$, Giovanni Otranto ${ }^{1}$, Claudio Ronco ${ }^{7}$
}

${ }^{1}$ U.O.C. Nefrologia e Dialisi, Ospedale Parodi-Delfino, Colleferro (RM)

${ }^{2}$ Divisione di Nefrologia, Ospedale S. Gerardo, Monza

${ }^{3}$ U.O.C. Nefrologia e Dialisi, Ospedale S. Paolo, Civitavecchia (RM)

${ }^{4}$ U.O.C. Nefrologia e Dialisi, ASST Lariana, Como

${ }^{5}$ U.O.C. Nefrologia e Dialisi, Ospedale V. Fazzi, Lecce

${ }^{6}$ Cattedra di Nefrologia, Università degli Studi Federico II, Napoli

${ }^{7}$ International Renal Research Institute, Ospedale S. Bortolo, Vicenza

\begin{abstract}
Galcetin - 3 and cardiorenal disease
Galectin-3 is a 32- to 35-kDa member of the galectin family of b-galactoside-binding lectins, which is characterized by a carbohydrate recognition domain. Through its carbohydrate-binding function, it regulates cell growth, differentiation, and inflammation. It also plays a complex, context-dependent role in the kidneys. During the development, it promotes nephrogenesis and is strongly expressed in the ureteric bud and its derivatives. An increase in the concentration of galectin-3 has been reported to be associated with fibrosis of the kidneys. Elevated levels of plasma galectin-3 are also associated with increased risks of rapid renal function decline, incident chronic kidney disease, and progressive renal impairment, and also with cardiovascular endpoints, infection, and all-cause mortality in patients with renal function impairment. This review discusses a general survey on galectin-3 expressions in nephrogenesis, kidney injury in animal models, clinical renal diseases, and renal transplantation and the potential role of galectin-3 for treatment in kidney disease.
\end{abstract}

Keywords: Acute kidney injury, Chronic kidney disease, Galectin-3, Heart failure, Nephrogenesis, Ventricular remodelling

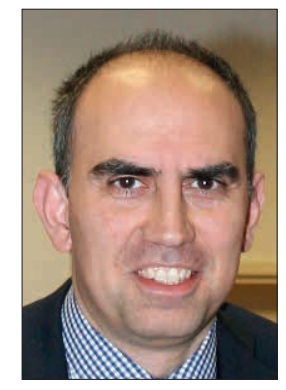

\section{Introduzione}

Le galectine costituiscono un gruppo proteico in grado di combinarsi per formare dei gruppi polisaccaridici attraverso dei processi di $\mathrm{N}$ - o di O-glicosilazione mediante un proprio dominio di riconoscimento specifico per i carboidrati (1-3).

Al momento attuale si riconoscono Luca Di Lullo 15 tipi diversi di galectine, le prime delle

Accepted: January 3, 2017

Published online: January 17, 2017

Indirizzo per la corrispondenza:

Dr. Luca Di Lullo

U.O.C. Nefrologia e Dialisi

Ospedale L. Parodi-Delfino

Piazza A. Moro 1

00034 Colleferro (RM)

dilulloluca69@gmail.com

quali isolate nel 1976, numerate in ordine cronologico e distribuite uniformemente in tutte le classi di organismi vertebrati $(1,2)$.

Allo stato attuale, la galectina-3 è l'unica dosabile nei mammiferi e si tratta di una molecola proteica dal peso molecolare di 32-35 kDa espressa a livello di cellule epiteliali ed endoteliali ma prodotta anche a livello dei macrofagi $(1,2)$.

La galectina-3 è in grado di modulare numerosi processi biologici mediante l'interazione con i suoi siti di legame con le molecole polisaccaridiche e non solo $(4,5)$.

Prodotta prevalentemente a livello citoplasmatico, essa può essere secreta all'esterno della cellula che la produce ma anche spostata all'interno dei nuclei cellulari.

La galectina-3 presente a livello extracellulare è in grado di modulare interazioni biologicamente significative quali quelle che avvengono tra cellule epiteliali e matrice extracellulare, giocando anche un ruolo nell'embriogenesi dei tubuli collettori renali (6).

Dall'altro lato, la galectina-3 presente a livello intracellulare è di fondamentale importanza per la sopravvivenza della cellula stessa grazie alla sua abilità nel bloccare la via intrinseca dell'apoptosi. 


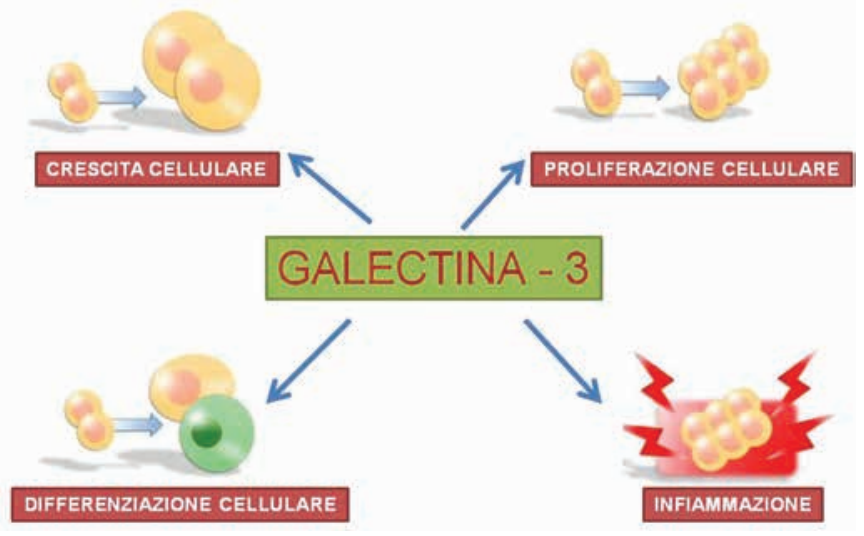

Fig. 1 - I ruoli fisiologici della galectina-3.

La quota di proteina presente all'interno dei nuclei, invece, sembra essere in grado di stimolare la proliferazione cellulare $(4,5)$.

Sono stati individuati diversi siti di legame per la galectina-3 a partire da numerose proteine glicosilate della matrice extracellulare (laminina, fibronectina e diverse integrine) (7).

I processi di adesione e proliferazione cellulare favoriti dalla presenza di galectina-3 possono, d'altro canto, favorire anche i processi di fibrosi cellulare e di progressione neoplastica.

In una serie di evidenze cliniche e sperimentali, la presenza di galectina-3 è stata associata a fibrosi cellulare, scompenso cardiaco, obesità, alterazioni del metabolismo glucidico e neoplasie (8-12).

La galectina-3, inoltre, è stata identificata con un ruolo attivo anche nella patogenesi del rimodellamento ventricolare sinistro, in diverse patologie di natura infettiva e in numerosi processi infiammatori e autoimmunitari $(9,13)$.

Nel danno tissutale acuto risulta essere un componente chiave nei meccanismi di difesa contro batteri come, per esempio, lo Streptococcus pneumoniae (14); la galectina-3, inoltre, regola il ciclo, la differenziazione e la proliferazione cellulare, nonché diversi processi di natura infiammatoria (Fig. 1).

La presente rassegna si prefigge lo scopo di presentare un quadro del ruolo della galectina-3 nella patologia cardiorenale con un focus particolare sulla nefrogenesi, sul danno renale acuto e cronico, sull'insufficienza cardiaca e sulla patologia cardiaca di natura ischemica.

\section{Il ruolo della galectina-3 nell'embriogenesi dell'apparato urinario}

La galectina-3 può essere dosata, con tecniche di immunofluorescenza, a livello delle cellule principali e di quelle intercalate dei tubuli collettori renali e, in misura minore, a livello delle cellule epiteliali del tratto ascendente dell'ansa di Henle (15). Nel corso dell'embriogenesi e, più precisamente, nel momento in cui si forma il cosiddetto "metanefro", la galectina-3 può essere dosata a livello delle porzioni apicali dell'uretere. Le quantità maggiori di galectina-3 sono documentabili, nel periodo fetale, nelle cellule epiteliali dei dotti collettori della midollare renale, sia a livello citoplasmatico che in sede di membrana basale. Livelli inferiori di galectina-3 sono stati riscontrati nel citoplasma di sottotipi cellulari dei tubuli collettori di soggetti adulti (16). La galectina-3 è in grado di influenzare la morfogenesi ureterale negli animali da esperimento ma non è, comunque, dosabile nei primi stadi dell'embriogenesi del tessuto renale. Negli stadi più avanzati della maturazione fetale, un ruolo prominente viene sicuramente esercitato nella maturazione definitiva dei dotti collettori della midollare renale (17).

L'espressione di galectina-3 è documentata fino agli stadi più avanzati della nefrogenesi laddove è principalmente coinvolta nella differenziazione finale del tessuto ureterale e dei tubuli distali $(6,18)$. In soggetti adulti normali, la produzione di galectina-3 è ristretta ai tubuli collettori e, solo parzialmente, ai tubuli distali, laddove le cellule epiteliali si trovino in una fase rigenerativa $(19,20)$.

\section{Galectina-3 e malattia renale}

\section{Galectina-3 e nefropatia diabetica}

La galectina-3 risulta andare incontro a un processo di up-regulation in modelli animali di nefropatia diabetica e di danno renale acuto (AKI, Acute Kidney Injury) (19-23). Alcuni Autori hanno valutato l'espressione di galectina-3 in biopsie renali di pazienti affetti da nefropatia diabetica, nefropatia a IgA, glomerulonefrite acuta con presenza di semilune e glomerulonefrite a lesioni minime.

Nei reni di soggetti normali, la presenza di galectina-3 è stata documentata a livello tubulare, ma non a livello glomerulare; nei soggetti affetti da nefropatia diabetica, la concentrazione di galectina-3 a livello glomerulare supera di gran lunga quella di qualunque altra tipologia di glomerulonefrite, con una percentuale decisamente elevata a livello dei macrofagi.

Nei pazienti diabetici è stata, inoltre, riscontrata una correlazione statisticamente significativa tra il numero delle cellule positive per la ricerca di galectina-3 a livello glomerulare e il tasso di compromissione della funzione renale (24).

Tali evidenze suggeriscono come l'infiltrazione di cellule ricche di galectina-3 possa giocare un ruolo determinante nella progressione della nefropatia diabetica soprattutto in termini prognostici.

\section{Galectina-3 e nefrite lupica}

Kang et al hanno esaminato 88 pazienti con nefrite lupica allo scopo di valutare un'eventuale associazione tra livelli di galectina-3 e stadio della malattia. La presenza di galectina-3 a livello glomerulare è stata documentata nell' $82 \%$ dei pazienti con nefrite lupica ma non nei soggetti controllo. I livelli 
di galectina-3 erano correlati con gli indici istologici di attività di malattia, con i livelli di C3 e C4, nonché con il titolo degli autoanticorpi anti dsDNA (25).

I pazienti affetti da nefrite lupica presentavano livelli sierici significativamente più elevati di galectina-3 con un'espressione decisamente aumentata a livello glomerulare, ad avvalorare l'ipotesi di come la galectina-3 sia in grado di modulare la risposta infiammatoria nei pazienti affetti da lupus eritematoso sistemico (25).

\section{Galectina-3 e malattia renale cronica (CKD)}

Come è ormai ben documentato, la malattia renale cronica (CKD, Chronic Kidney Disease) si configura come una questione di salute pubblica a livello mondiale, visti i numeri drammaticamente in ascesa e visto, soprattutto, l'elevato tasso di morbidità e mortalità per malattia cardiovascolare in questa popolazione di pazienti (26-30).

Allo stesso tempo, la progressione della malattia renale può essere rallentata $\mathrm{o}$, addirittura, bloccata nel momento in cui si riesca a porre una diagnosi precoce la quale consente di evitare le pericolose conseguenze nelle quali si rischia di incorrere (31).

Diventa, quindi, importante il dosaggio di biomarcatori in grado di consentire l'identificazione di soggetti a rischio già dai primissimi stadi di malattia.

I livelli sierici di galectina-3, per esempio, sembra siano correlati allo sviluppo di fibrosi renale in animali da esperimento e sembrano essere correlati, in modo inversamente proporzionale, con il valore di eGFR nella popolazione umana adulta (32); ciò che rimane ancora da determinare è l'eventuale ruolo predittivo dei livelli di galectina- 3 in caso di malattia renale.

Un gruppo di ricercatori ha esaminato i dati provenienti da 2450 pazienti dello studio di Framingham e ne ha valutato gli outcome renali in un periodo medio di follow-up di circa 10 anni (33). Dall'analisi dei suddetti dati è risultato come vi sia stato un declino del filtrato glomerulare (eGFR $>3 \mathrm{~mL} / \mathrm{min} /$ $1.73 \mathrm{~m}^{2} / \mathrm{anno}$ ) nel $9.2 \%$ dei pazienti, con un'incidenza di CKD (eGFR $<60 \mathrm{~mL} / \mathrm{min} / 1.73 \mathrm{~m}^{2} / \mathrm{anno}$ ) e di albuminuria (rapporto albuminuria/creatininuria $>17 \mathrm{mg} / \mathrm{g}$ negli uomini e $>25 \mathrm{mg} / \mathrm{g}$ nelle donne) pari, rispettivamente, all'11.3\% e al $10.1 \%$. Nello stesso report, inoltre, sono state documentate un'associazione significativa tra i livelli più elevati di galectina-3 e un più rapido deterioramento della funzione renale così come una maggiore incidenza (nuovi casi) di CKD; allo stesso tempo, non vi era nessuna associazione tra livelli di galectina-3 e grado di albuminuria (33).

Nei primi stadi di malattia renale cronica, la presenza di fibrosi tubulo-interstiziale (per la quale non esiste un corrispettivo dal punto di vista clinico) diventa importante per una diagnosi precoce di nefropatia ma, al contempo, la mancata associazione tra livelli di galectina-3 e albuminuria contraddice l'ipotesi che il danno glomerulare o l'assottigliamento dei podociti possano essere considerati parte dei meccanismi fisiopatologici a monte del danno renale stesso.

Il fatto che livelli plasmatici più elevati di galectina-3 siano associati a un rischio più elevato di CKD incidente e di declino della funzione renale ha suggerito l'ipotesi che il dosaggio dei livelli sierici di galectina-3 potesse essere utile per la diagnosi precoce del danno renale ancora prima che si possa sviluppare un quadro di malattia renale clinicamente conclamata ma quest'ipotesi richiede, allo stato attuale, ulteriori verifiche.

Indubbiamente la galectina-3 gioca un ruolo di primo piano nella fase di esordio e di mantenimento della nefropatia diabetica e non (34).

Impiegando un modello animale nel quale si veniva a creare un'ostruzione ureterale monolaterale, la distruzione genetica del gene codificante per la galectina- 3 consentiva di attenuare il grado di fibrosi renale (22).

Alcuni Autori hanno analizzato due gruppi numerosi di pazienti arruolati negli studi 4D (1168 pazienti affetti da diabete mellito di tipo 2 sottoposti a trattamento emodialitico) e LURIC (2579 pazienti affetti da cardiopatia ischemica e sottoposti a esame coronarografico) e caratterizzati da livelli di funzione renale quanto mai eterogenei (35). Le concentrazioni di galectina-3 venivano dosate al tempo zero e i pazienti venivano stratificati in tre gruppi in base ai livelli di filtrato glomerulare: $>90$, tra 60 e 89 e $<60 \mathrm{~mL} / \mathrm{min} / 1.73 \mathrm{~m}^{2}$.

I risultati hanno evidenziato come i livelli di galectina-3 non fossero associati a outcome a lungo termine nel gruppo di pazienti con eGFR $>90 \mathrm{~mL} / \mathrm{min}$ dello studio LURIC, bensì al tasso di mortalità per tutte le cause, per cause cardiovascolari, per cause infettive e per morte cardiaca improvvisa nei pazienti con livelli di eGFR compresi tra 60 e $89 \mathrm{~mL} / \mathrm{min}$.

Nei pazienti con eGFR $<60 \mathrm{~mL} / \mathrm{min}$, la concentrazione di galectina-3 era, inoltre, associata a infarto del miocardio e a morte per scompenso cardiaco cronico. Nella popolazione di pazienti del 4D, i livelli di galectina-3 possedevano un valore predittivo in termini di mortalità per tutte le cause, eventi cardiovascolari maggiori, ictus cerebrale e mortalità da patologia infettiva (35).

\section{Galectina-3 e trapianto renale}

Sebbene il numero crescente di trapianti renali abbia positivamente influito sulla sopravvivenza e sul miglioramento della qualità di vita dei pazienti con malattia renale croni$\mathrm{ca}$, la durata del trapianto stesso resta ancora una sfida da vincere (36).

I meccanismi fisiopatologici in grado di determinare un rigetto cronico non sono stati ancora del tutto chiariti, così come i fattori di rischio patognomonici dello stesso paziente trapiantato. Per i motivi appena citati diventa imperativo cercare di identificare dei biomarcatori in grado di predire un eventuale rigetto e di implementare delle strategie terapeutiche che possano consentire un aumento della durata media di vita del rene trapiantato. 


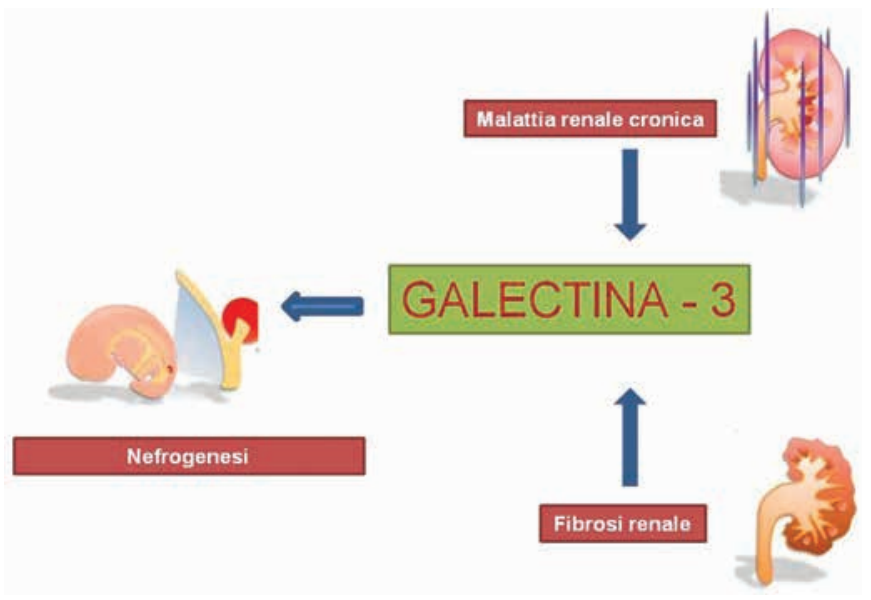

Fig. 2 - II ruolo della galectina-3 nella malattia renale cronica.

Nei paragrafi precedenti si è parlato del ruolo della galectina-3 come potenziale biomarcatore di malattia renale $(22,34)$ ma poco si sa dell'espressione di galectina-3 nei pazienti con trapianto renale.

Uno studio abbastanza recente ha voluto esaminare i livelli sierici di galectina-3 in una coorte di pazienti sottoposti a trattamento emodialitico e in un'altra di pazienti trapiantati (37). Lo studio ha arruolato 41 soggetti sani, 41 portatori di trapianto renale e 32 in trattamento emodialitico e i primi risultati hanno permesso di evidenziare livelli di galectina-3 più elevati nel gruppo di pazienti trapiantati e in quello dei soggetti sottoposti a emodialisi. In un'analisi successiva fatta dopo tre mesi, si è evidenziato un calo dei livelli di galectina-3 nei pazienti trapiantati, mentre nulla si modificava nei pazienti sottoposti a trattamento emodialitico (37).

Da quanto emerso sembrerebbe evidente che il trapianto renale comporta una limitazione dell'espressione dei geni codificanti per la galectina-3, anche se ci sarebbe da studiare il ruolo esercitato, a livello genico, dalla concomitante terapia immunosoppressiva.

In studi sperimentali si è, inoltre, documentato un ruolo attivo della galectina-3 nel promuovere la fibrosi renale nel rene trapiantato, suggerendo come essa possa diventare un bersaglio terapeutico nella prevenzione del rigetto cronico (38).

\section{Galectina-3 e nuove opportunità terapeutiche nella malattia renale cronica}

Da quanto discusso nelle sezioni precedenti, risulta evidente come un incremento della concentrazione di galectina-3 sia associato a fibrosi renale e a un incremento del rischio di riduzione della funzione renale e di nuovi casi di malattia renale cronica (Fig. 2). La galectina-3, inoltre, sembra giocare un ruolo di primo piano nella modulazione di diverse risposte infiammatorie con un aumento delle concentrazioni sieriche in risposta a un danno renale di natura ischemica e/o tossica (19).

Inoltre, sembra essere in grado di prevenire un danno tubulare cronico riducendo il grado di apoptosi cellulare e favorendo il rimodellamento delle matrici extracellulari (39); le proprietà reno-protettive della galectina-3 sono, inoltre, testimoniate dal rapido peggioramento della nefropatia diabetica in ratti nei quali veniva soppresso il gene codificante per la proteina stessa (24).

Allo stesso tempo, in presenza di un danno tissutale persistente o ripetuto, la galectina- 3 è in grado di modulare la transizione verso una condizione di infiammazione cronica e fibrosi (9).

La galectina-3 è un potente attivatore dei fibroblasti a livello renale ed è responsabile dello sviluppo di fibrosi nei soggetti portatori di trapianto renale che vanno incontro a fenomeni di rigetto cronico (38).

Uno studio di Sun et al ha analizzato gli effetti dei prodotti finali della degradazione dei glucidi (AGE, Advanced Glycation End-product) e della terapia con rosiglitazone sull'espressione dei geni per la galectina-3 in colture di cellule mesangiali umane; il rosiglitazone è risultato in grado di incrementare l'espressione e la secrezione di galectina-3 in modo dose-dipendente, suggerendo come la molecola possa giocare un ruolo nella nefroprotezione galectina-3 mediata (40).

Sebbene sia ormai documentata l'aumentata secrezione di galectina-3 in corso di danno renale acuto, l'importanza di questa iperincrezione è tutta da decifrare.

Alcuni Autori hanno analizzato le modificazioni dell'espressione di galectina-3 in topi con danno renale acuto indotto da acido folico impiegando un derivato della pectina (MCP, Modified Citrus Pectin) in grado di legarsi al dominio di riconoscimento dei carboidrati della molecola di galectina-3. La somministrazione di MCP ha inciso negativamente sulla proliferazione cellulare renale senza, al contempo, influire sul grado di apoptosi cellulare. Dopo due settimane, i topi trattati con MCP hanno evidenziato una riduzione dei livelli di galectina-3, del grado di fibrosi renale, della percentuale di apoptosi e dell'espressione di citochine ad azione pro-infiammatoria da parte dei macrofagi. Tali evidenze supportano l'azione protettiva della MCP sull'evoluzione della nefropatia grazie a un'azione che modula la proliferazione cellulare in fase precoce e, a lungo termine, su espressione di galectina-3, grado di fibrosi e tasso di apoptosi (41).

Di conseguenza, MCP potrebbe configurarsi come un nuovo bersaglio sul quale concentrarsi per ridurre il danno renale a lungo termine, probabilmente grazie alla mediazione dei siti di legame della galectina-3 (41).

In definitiva, quindi, i livelli di galectina-3 possono fornire indicazioni in termini di prognosi e di possibili strategie terapeutiche in chiave anti-galectina-3, ma il tutto necessita di ulteriori studi di natura prospettica. 


\section{Galectina-3 e scompenso cardiaco}

\section{Galectina-3 e fibrosi cardiaca}

In soggetti adulti sani, i livelli miocardici di galectina-3 sono estremamente ridotti ma tendono ad aumentare in coloro i quali sono affetti da scompenso cardiaco congestizio, assumendo, quindi, un ruolo di natura prognostica (42). Di qui è stato proposto un possibile coinvolgimento della galectina-3 nei meccanismi fisiopatologici in grado di condurre a fibrosi miocardica. Un'aumentata produzione di galectina-3 è stata dimostrata in pazienti con stenosi valvolare aortica (che evidenziano un quadro di ipertrofia ventricolare sinistra) e nelle cardiomiopatie indotte, in animali da laboratorio, da interferone e streptozotocina.

In questi quadri clinici/sperimentali, i cardiomiociti esprimono quantità praticamente indosabili di galectina-3, mentre i fibroblasti localizzati a livello miocardico ne sono ricchi (43). Analisi immunoistochimiche effettuate su topi con cardiomiopatia ipertrofica hanno evidenziato una prevalente localizzazione di galectina-3 a livello di fibroblasti, macrofagi e matrice extracellulare (43). È stato, inoltre, evidenziato come in modelli sperimentali di miocardite e cardiomiopatia ipertrofica vi sia una cospicua infiltrazione miocardica di macrofagi attivati ricchi di galectina-3, a testimonianza del ruolo della stessa nel processo infiammatorio $(43,44)$.

L'insufficienza cardiaca si caratterizza per essere una sindrome complessa con diversi meccanismi fisiopatologici che sottintendono l'insorgenza della stessa; tra questi un ruolo di primo piano sembra essere giocato dai meccanismi infiammatori (45).

Nel momento in cui si determina un danno di qualsiasi natura in sede miocardica, si assiste al reclutamento in situ di macrofagi e altre cellule immunitarie in grado di iniziare il processo riparativo (45).

Diversi studi suggeriscono un legame evidente tra attivazione dei macrofagi e fibrosi nella patogenesi dell'insufficienza cardiaca (46); la galectina-3 viene secreta a livello extra-cellulare dai macrofagi, grazie anche alla mediazione dell'osteopontina, proprio nel punto in cui si è verificato il danno miocardico (42). In conseguenza di ciò, si assiste a un'attivazione dei fibroblasti a livello della matrice extra-cellulare, mentre la secrezione contemporanea di galectina-3 promuove la produzione di citochine ad azione pro-infiammatoria come il TGF (Transforming Growth Factor)- $\beta$ (47).

In conseguenza di quanto appena descritto, si determina un'attivazione dei fibroblasti a livello miocardico con produzione e deposizione di collagene di tipo I a livello extra-cellulare: il risultato è quello, a livello clinico, di una disfunzione sisto-diastolica del ventricolo sinistro (43).

L'attivazione dei fibroblasti è, inoltre, caratterizzata, dall'aumentata espressione di proteine strutturali del citoscheletro cellulare come actina e collagene di tipo I, il tutto indotto dall'aumentata secrezione di galectina-3 (42), la quale, in più, contribuisce all'inibizione della degradazione della matrice extra-cellulare, incrementando il grado di fibrosi miocardica $(42,48)$.

Livelli elevati di galectina-3 comportano il rilascio di diversi mediatori (vedi TGF- $\beta$ ) in grado di favorire la proliferazione dei fibroblasti e la deposizione di fibre collagene con conseguente disfunzione diastolica del ventricolo sinistro (43).

Un aumento della produzione di galectina-3 è stato documentato anche nello stadio di ipertrofia concentrica in fase di compenso, preludio al quadro di scompenso cardiaco congestizio (49), a testimonianza del fatto che il pattern istologico di fibrosi miocardica precede lo sviluppo dei sintomi clinici di insufficienza cardiaca.

\section{Galectina-3 e rimodellamento cardiaco}

La fibrosi miocardica è uno dei meccanismi fisiopatologici che sottintendono il rimodellamento cardiaco in corso di insufficienza cardiaca ed è, come già descritto in precedenza, un processo caratterizzato dall'accumulo di fibroblasti e dal deposito di proteine della matrice extra-cellulare in risposta a un processo infiammatorio che, alla lunga, comporta una modificazione dell'architettura, della struttura e della funzione del tessuto miocardico (50). In questo contesto clinico, un ruolo chiave è giocato dai macrofagi, come dimostrato dal fatto che una delezione specifica di questa popolazione cellulare comporta la riduzione dell'attivazione dei fibroblasti presenti a livello miocardico e del conseguente grado di fibrosi (51).

Di concerto con l'azione esercitata dai macrofagi, la secrezione di galectina-3 contribuisce in larga misura al reclutamento/attivazione dei fibroblasti con conseguente sviluppo di una fibrosi miocardica (42).

Come a livello renale, anche in sede miocardica la galectina-3 modula la risposta infiammatoria e immunitaria ed è coinvolta nel quadro di ipertrofia ventricolare sinistra (43).

I livelli di galectina-3 sono significativamente correlati alle modificazioni del volume telediastolico del ventricolo sinistro, la cui riduzione si associa a livelli inferiori di galectina-3 (52).

È stato, inoltre, dimostrato come i livelli di galectina-3 siano correlati significativamente con il grado di ipertrofia ventricolare sinistra in pazienti affetti da ipertensione arteriosa o diabete mellito in trattamento emodialitico. Più in particolare, le concentrazioni più elevate di galectina-3 sono state documentate sia in pazienti con ipertrofia concentrica che in pazienti con ipertrofia eccentrica del ventricolo sinistro (53).

\section{Il ruolo clinico della galectina-3 in corso di insufficienza cardiaca}

\section{Galectina-3: un fattore di rischio per insufficienza cardiaca acuta e cronica}

Alla luce di quanto osservato in studi sperimentali, sono stati disegnati diversi trial clinici allo scopo (a) di evidenziare 
il valore diagnostico e prognostico dei livelli di galectina-3 in base ai suoi livelli plasmatici e (b) di personalizzare eventuali interventi terapeutici e monitorare la risposta alla terapia.

Il valore prognostico della galectina-3 è stato evidenziato in trial clinici come l'HF_ACTION e il $\mathrm{COACH}$, nei quali livelli sierici superiori a $17 \mathrm{ng} / \mathrm{mL}$ erano associati a una più rapida progressione del quadro di insufficienza cardiaca, come testimoniato dal numero delle ospedalizzazioni e dei decessi registrati $(54,55)$.

Alla luce delle suddette evidenze è stato proposto che la galectina-3 non sia soltanto un biomarcatore di fibrosi miocardica, bensì un mediatore nella progressione del quadro di insufficienza cardiaca.

II ruolo della galectina- 3 come indice prognostico (a breve termine) è stato valutato nello studio PRIDE, dove venivano valutati i pazienti giunti in reparto di medicina d'urgenza per dispnea ingravescente (56).

Dei 209 pazienti con scompenso cardiaco acuto valutati, il $29 \%$ aveva presentato una recidiva di scompenso cardiaco e l'8\% era deceduto nel periodo d'osservazione (56).

In questi ultimi due gruppi di pazienti la concentrazione media di galectina-3 era superiore a $9.42 \mathrm{ng} / \mathrm{mL}$ (56).

In un'analisi post-hoc dello studio multicentrico ADHF, pazienti valutati a un anno dall'arruolamento nello studio con livelli sierici di galectina- 3 inferiori a $12 \mathrm{ng} / \mathrm{mL}$ presentavano un tasso di mortalità del $10.3 \%$ rispetto al tasso del $35 \%$ dei pazienti con livelli sierici di galectina-3 superiori a $22.3 \mathrm{ng} / \mathrm{mL}$ (57).

II valore prognostico a lungo termine della galectina- 3 è stato valutato nello studio $\mathrm{COACH}$ (periodo di follow-up di 18 mesi), dove venivano arruolati pazienti affetti da scompenso cardiaco cronico. I risultati ottenuti hanno evidenziato il ruolo della galectina-3 quale fattore prognostico per mortalità per tutte le cause e per rischio di nuove ospedalizzazioni in pazienti affetti da scompenso cardiaco cronico (55).

\section{Galectina-3 e insufficienza cardiaca con funzione sistolica preservata}

De Boer et al hanno evidenziato, in uno studio relativamente recente, come i livelli sierici di galectina-3 non fossero significativamente diversi in pazienti con scompenso cardiaco e funzione sistolica del ventricolo sinistro conservata (frazione d'eiezione $>40 \%$ ) o con funzione sistolica ridotta (frazione d'eiezione $<40 \%$ ), anche se il valore prognostico presenta un impatto maggiore nei soggetti con frazione d'eiezione conservata (58).

II quadro fisiopatologico dello scompenso cardiaco con funzione sistolica conservata è caratterizzato dalla presenza di ipertrofia, deposizione di matrice extracellulare e rigidità delle fibre miocardiche, motivo per il quale l'importanza della galectina-3 come biomarcatore di fibrosi sembra essere indiscutibile $(59,60)$.

La galectina-3 è un biomarcatore che, riflettendo l'entità di un processo fibrotico e infiammatorio, ben si presta a un suo impiego come fattore predittivo per sviluppo di insufficienza cardiaca e per l'eventuale evoluzione sfavorevole di un quadro di scompenso cardiaco con funzione sistolica conservata $(61,58)$.

Sia nell'analisi post-hoc dello studio di Framingham sia nello studio PREVEND, per un totale di circa 10.000 pazienti, i livelli plasmatici di galectina-3 presentavano una forte associazione con i livelli di pressione arteriosa $(61,62)$. La pressione arteriosa gioca un ruolo fondamentale nella genesi dell'insufficienza cardiaca con funzione sistolica preservata e l'associazione appena descritta può offrire la chiave della correlazione tra scompenso cardiaco con funzione sistolica conservata e livelli sierici di galectina-3 (63).

Un altro fattore prognostico da considerare è quello legato alla presenza di diabete mellito, fattore prognostico sfavorevole spesso presente nella quota di pazienti affetti da scompenso cardiaco con funzione sistolica conservata. Nella popolazione generale e in quella di pazienti a rischio di sviluppo della suddetta forma di insufficienza cardiaca, il diabete risulta associato a livelli sierici aumentati di galectina-3 (63).

La galectina-3 è stata, inoltre, individuata come importante mediatore nella rimozione dei prodotti finali della glicosilazione derivati dall'ossidazione e dalla glicosilazione non enzimatica delle proteine (64). Tali prodotti della glicosilazione si accumulano a livello miocardico e contribuiscono all'aumento della rigidità (stiffness) delle fibre miocardiche (65).

Livelli elevati di galectina-3 sono, inoltre, documentabili nei pazienti obesi e l'obesità è uno dei fattori di comorbidità nei pazienti affetti da scompenso cardiaco con funzione sistolica preservata (66).

Ipertensione arteriosa, diabete e obesità rappresentano fattori di rischio responsabili dell'insorgenza di fibrillazione atriale, condizione clinica caratterizzata, a livello istologico, dalla presenza di un'estesa fibrosi atriale (67); in uno studio di Clementy et al, elevati livelli sierici di galectina-3 erano correlati alla presenza di una fibrillazione atriale persistente ma non a episodi di parossismo, elemento utile per la selezione di pazienti in grado di beneficiare dell'eventuale terapia di ablazione (67).

\section{Galectina-3: Un Potenziale Fattore Di Correlazione Tra Scompenso Cardiaco E Malattia Renale Cronica?}

I livelli sierici di galectina-3 risultano essere, dunque, incrementati sia nei pazienti affetti da scompenso cardiaco acuto (risultando ancora più elevati nei pazienti in classe NYHA III-IV e con prognosi peggiore) sia in quelli affetti da malattia renale cronica $(57,58)$.

Nei pazienti con scompenso cardiaco sia acuto che cronico, livelli elevati di galectina-3 sono stati associati alla presenza di alterazioni della funzione renale e a tassi elevati di mortalità per tutte le cause $(57,68)$.

Tang et al hanno documentato un'associazione significativa tra livelli sierici di galectina-3 e malattia renale grazie al dosaggio contemporaneo della cistatina C (32). 
Inoltre, livelli elevati di galectina-3 possono essere associati a un peggioramento del quadro cardiaco non solo per un meccanismo diretto di tipo cardiotossico, ma anche per interessamento del cosiddetto asse cardio-renale.

Sia nello studio PRIDE che in quello DEAL-HF si è osservata una stretta correlazione tra concentrazioni di galectina-3 e livello di eGFR $(56,68)$.

Il valore prognostico del dosaggio di galectina-3 nello scompenso cardiaco può in parte riflettere le conseguenze di un quadro di disfunzione renale, come documentato dalla relazione inversa esistente tra livelli di galectina-3 e parametri di funzione renale in pazienti con scompenso cardiaco; questa evidenza supporterebbe l'ipotesi che livelli elevati di galectina-3 in questi pazienti sarebbero legati all'alterazione della funzione renale piuttosto che al quadro di insufficienza cardiaca (69).

Sono state proposte diverse ipotesi per spiegare la presenza di elevati livelli di galectina-3 nei pazienti con malattia renale cronica: dalla possibilità di considerarla un biomarcatore di ridotta funzione renale per le sue proprietà di clearance renale stessa alla potenziale iperincrezione renale per motivi tutti da decifrare, fino al suo effetto pro-fibrotico a livello glomerulare e tubulo-interstiziale (70).

Infine, va detto come sia una condizione di insufficienza cardiaca che la presenza di un quadro di malattia renale cronica possano indurre uno stato di infiammazione sistemica in grado di stimolare la produzione di galectina- 3 anche in altri organi che non siano il cuore o il rene $(70,71)$.

\section{Conclusioni}

Alla luce di quanto discusso nella presente rassegna, risulta evidente come la galectina-3 sia chiaramente coinvolta nelle dinamiche fisiopatologiche che sottintendono lo sviluppo di un quadro di scompenso cardiaco o di malattia renale cronica.

Il suo valore predittivo, in termini di outcome clinici sfavorevoli, è stato evidenziato in numerosi studi clinici e sperimentali, sia come biomarcatore "stand-alone" sia in associazione con altre molecole.

Un ruolo fondamentale è, senza dubbio, quello ricoperto nell'ambito dello scompenso cardiaco con funzione sistolica preservata, condizione clinica caratterizzata da un elevato grado di fibrosi.

Dati meno rigorosi sono quelli relativi al fatto che i livelli sierici di galectina-3 possano essere influenzati o derivare da un quadro di riduzione della funzione renale, comorbidità spesso presente nei pazienti affetti da insufficienza cardiaca.

Infine, esistono evidenze molto promettenti in merito al fatto che la galectina-3 potrebbe diventare il bersaglio per trattamenti farmacologici in grado di bloccarne l'attività (per esempio, con i derivati della pectina) e modificare la storia clinica dei pazienti con patologia cardio-renale.

\section{Disclosures}

Financial support: No financial support was received for this submission. Conflict of interest: The authors have no conflict of interest.

\section{Bibliografia}

1. Barondes SH, Cooper DN, Gitt MA, Leffler H. Galectins. Structure and function of a large family of animal lectins. J Biol Chem. 1994;269:20807-20810.

2. Cummings RD, Liu FT. Galectins. In Essentials of Glycobiology, 2nd ed.; Varki A, Cummings RD, Esko JD, Freeze HH, Stanley P, Bertozzi CR, Hart GW, Etzler ME, eds.; Cold Spring Harbor Laboratory Press: Cold Spring Harbor, NY, USA. 2009.

3. Kasai K, Hirabayashi J. Galectins: A family of animal lectins that decipher glycocodes. J Biochem. 1996;119:1-8.

4. Davidson PJ, Davis MJ, Patterson RJ, Ripoche MA, Poirier F, Wang JL. Shuttling of galectin-3 between the nucleus and cytoplasm. Glycobiology. 2002;12:329-937.

5. Hsu DK, Liu FT. Regulation of cellular homeostasis by galectins. Glycoconj J. 2004;19:507-515.

6. Ochieng J, Furtak V, Lukyanov P. Extracellular functions of galectin-3. Glycoconj J. 2004;19:527-535.

7. Dumic J, Dabelic S, Flogel M. Galectin-3: An open-ended story. Biochim Biophys Acta. 2006;1760:616-635.

8. De Boer RA, Voors AA, Muntendam P, van Gilst WH, van Veldhuisen DJ. Galectin-3: A novel mediator of heart failure development and progression. Eur J Heart Fail. 2009;11:811-817.

9. Henderson NC, Sethi T. The regulation of inflammation by galectin-3. Immunol Rev. 2009;230:160-171.

10. Sakaki M, Fukumori T, Fukawa T, et al. Clinical significance of Galectin-3 in clear cell renal cell carcinoma. J Med Investig. 2010;57:152-157.

11. Straube T, Elli AF, Greb C, et al. Changes in the expression and subcellular distribution of galectin-3 in clear cell renal cell carcinoma. J Exp Clin Cancer Res. 2011;30:89.

12. Menini S, lacobini C, Blasetti Fantauzzi C, Pesce CM, Pugliese G. Role of galectin-3 in obesity and impaired glucose homeostasis. Oxid Med Cell Longev. 2016;2016:9618092.

13. Dhirapong A, Lleo A, Leung P, Gershwin ME, Liu FT. The immunological potential of galectin-1 and -3 . Autoimmun Rev. 2009;8:360-363.

14. Feldman C. Clinical relevance of antimicrobial resistance in the management of pneumococcal community-acquired pneumonia. J Lab Clin Med. 2004;143:269-283.

15. Bichara M, Attmane-Elakeb A, Brown D, et al. Exploring the role of galectin 3 in kidney function: A genetic approach. Glycobiology. 2006;16:36-45

16. Winyard PJ, Bao Q, Hughes RC, Woolf AS. Epithelial galectin-3 during human nephrogenesis and childhood cystic diseases. J Am Soc Nephrol. 1997;8:1647-1657.

17. Bullock SL, Johnson TM, Bao Q, Hughes RC, Winyard PJ, Woolf AS. Galectin-3 modulates ureteric bud branching in organ culture of the developing mouse kidney. J Am Soc Nephrol. 2001;12:515-523.

18. Iacobini C, Menini S, Oddi G, et al. Galectin-3/AGE-receptor 3 knockout mice show accelerated AGE-induced glomerular injury: Evidence for a protective role of galectin-3 as an $A G E$ receptor. FASEB J. 2004;18:1773-1775.

19. Nishiyama J, Kobayashi S, Ishida A, et al. Up-regulation of galectin-3 in acute renal failure of the rat. Am J Pathol. 2000; 157:815-823.

20. Lohr M, Kaltner H, Lensch M, Andre S, Sinowatz F, Gabius HJ. Celltype-specific expression of murine multifunctional galectin-3 and its association with follicular atresia/luteolysis in contrast to 
pro-apoptotic galectins-1 and -7. Histochem Cell Biol. 2008;130: 567-581.

21. Diamond JR. Macrophages and progressive renal disease in experimental hydronephrosis. Am J Kidney Dis. 1995;26:133-140.

22. Henderson NC, Mackinnon AC, Farnworth SL, et al. Galectin-3 expression and secretion links macrophages to the promotion of renal fibrosis. Am J Pathol. 2008;172:288-298.

23. Pugliese $G$, Pricci $F$, lacobini $C$, et al. Accelerated diabetic glomerulopathy in galectin-3/AGE receptor 3 knockout mice. FASEB J. 2001;15:2471-2479.

24. Kikuchi Y, Kobayashi S, Hemmi N, et al. Galectin-3-positive cell infiltration in human diabetic nephropathy. Nephrol Dial Transplant. 2004;19:602-607.

25. Kang EH, Moon KC, Lee EY, et al. Renal expression of galectin-3 in systemic lupus erythematosus patients with nephritis. Lupus. 2009;18:22-28.

26. Ensrud KE, Lui LY, Taylor BC, et al. Renal function and risk of hip and vertebral fractures in older women. Arch Intern Med. 2007;167:133-139.

27. McClellan WM, Flanders WD. Risk factors for progressive chronic kidney disease. J Am Soc Nephrol. 2003;14:S65-S70.

28. Perkovic V, Cass A, Patel AA, et al. High prevalence of chronic kidney disease in Thailand. Kidney Int. 2008;73:473-479.

29. Tanaka H, Shiohira Y, Uezu Y, Higa A, Iseki, K. Metabolic syndrome and chronic kidney disease in Okinawa, Japan. Kidney Int. 2006;69:369-374.

30. Weiner DE, Tighiouart H, Amin MG, et al. Chronic kidney disease as a risk factor for cardiovascular disease and all-cause mortality: A pooled analysis of community-based studies. J Am Soc Nephrol. 2004;15:1307-1315.

31. De Zeeuw D, Ramjit D, Zhang Z, et al. Renal risk and renoprotection among ethnic groups with type 2 diabetic nephropathy: A post hoc analysis of RENAAL. Kidney Int. 2006;69: 1675-1682.

32. Tang WH, Shrestha K, Shao Z, et al. Usefulness of plasma galectin-3 levels in systolic heart failure to predict renal insufficiency and survival. Am J Cardiol. 2011;108:385-390.

33. O'Seaghdha CM, Hwang SJ, Ho JE, Vasan RS, Levy D, Fox CS. Elevated galectin-3 precedes the development of CKD. J Am Soc Nephrol. 2013;24:1470-1477.

34. Iacobini $\mathrm{C}$, Amadio $\mathrm{L}$, Oddi $\mathrm{G}$, et al. Role of galectin-3 in diabetic nephropathy. J Am Soc Nephrol. 2003;14:S264-S270.

35. Drechsler C, Delgado G, Wanner C, et al. Galectin-3, renal function, and clinical outcomes: Results from the LURIC and 4D studies. J Am Soc Nephrol. 2015;26:2213-2221.

36. Arend SM, Mallat MJ, Westendorp RJ, van der Woude FJ, van Es LA. Patient survival after renal transplantation; more than 25 years follow-up. Nephrol Dial Transplant. 1997;12:1672-1679.

37. Tan R, Liu X, Wang J, et al. Alternations of galectin levels after renal transplantation. Clin Biochem. 2014;47:83-88.

38. Dang Z, MacKinnon A, Marson LP, Sethi T. Tubular atrophy and interstitial fibrosis after renal transplantation is dependent on galectin-3. Transplantation. 2012;93:477-484.

39. Okamura DM, Pasichnyk K, Lopez-Guisa JM, et al. Galectin-3 preserves renal tubules and modulates extracellular matrix remodeling in progressive fibrosis. Am J Physiol Ren Physiol. 2011;300:F245-253.

40. Sun ZL, Ma CJ, Jin H, Yuan Y, Liu NF. Effects of advanced glycosylation end products and rosiglitazone on the expression and secretion of galectin-3 in human renal mesangial cells. Chin Med J. 2009;122:1067-1071.

41. Kolatsi-Joannou M, Price KL, Winyard PJ, Long DA. Modified citrus pectin reduces galectin-3 expression and disease severity in experimental acute kidney injury. PLoS ONE. 2011;6:e18683.

42. De Boer RA, Voors AA, Muntendam P, van Gilst WH, van Veld- huisen DJ. Galectin-3: a novel mediator of heart failure development and progression. Eur J Heart Fail. 2009;11:811-817.

43. Sharma UC, Pokharel S, Van Brakel TJ, et al. Galectin-3 marks activated macrophages in failure-prone hypertrophied hearts and contributes to cardiac dysfunction. Circulation. 2004; 110:3121-3128.

44. Reifenberg K, Lehr HA, Torzewski M, et al. Interferon- gamma induces chronic active myocarditis and cardiomyopathy in transgenic mice. Am J Pathol. 2007;171:463-472.

45. Oikonomou E, Tousoulis D, Siasos G, Zaromitidou M, Papavassiliou AG, Stefanadis C. The role of inflammation in heart failure: new therapeutic approaches. Hellenic J Cardiol. 2011;52:30-40.

46. Weber KT, Gerling IC, Kiani MF, et al. Aldosteronism in heart failure: a proinflammatory/fibrogenic cardiac phenotype. Search for biomarkers and potential drug targets. Curr Drug Targets. 2003;4:505-516.

47. Psarras S, Mavroidis M, Sanoudou D, et al. Regulation of adverse remodelling by osteopontin in a genetic heart failure model. Eur Heart J. 2012;33:1954-1963.

48. Lin YH, Lin LY, Wu YW, et al. The relationship between serum galectin-3 and serum markers of cardiac extracellular matrix turnover in heart failure patients. Clin Chim Acta. 2009;409: 96-99.

49. Sharma UC, Pokharel S, van Brakel TJ, et al. Galectin-3 marks activated macrophages in failure-prone hypertrophied hearts and contributes to cardiac dysfunction. Circulation. 2004;110: 3121-3128.

50. Krenning G, Zeisberg EM, Kalluri R. The origin of fibroblasts and mechanism of cardiac fibrosis. J Cell Physiol. 2010;225:631-637.

51. Henderson NC, Mackinnon AC, Farnworth SL, et al. Galectin-3 expression and secretion links macrophages to the promotion of renal fibrosis. Am J Pathol. 2008;172:288-298.

52. Lok DJ, Lok SI, Bruggink-André de la Porte PW, et al. Galectin-3 is an independent marker for ventricular remodeling and mortality in patients with chronic heart failure. Clin Res Cardiol. 2012;102:103-110.

53. Yilmaz H, Gurel OM, Celik HT, et al. Relationship of galectin-3 to left ventricular geometry and hypertrophy in chronic hemodialysis patients. Herz. 2015;40 (4): 702-708.

54. Felker GM, Fiuzat M, Shaw LK, et al. Galectin-3 in ambulatory patients with heart failure: results from the HF-ACTION study. Circ Heart Fail. 2012;5:72-78.

55. De Boer RA, Lok D, Hillege JL, et al. Clinical and prognostic value of galectin-3, a novel fibrosis-associated biomarker. Relation with clinical and biochemical correlates of heart failure. J Am Coll Cardiol. 2010;55:A26.

56. Januzzi JL Jr, Camargo CA, Anwaruddin S, et al. The N-terminal Pro-BNP investigation of dyspnea in the emergency department (PRIDE) study. Am J Cardiol. 2005;95:948-954.

57. Van Kimmenade RR, Januzzi JL Jr, Ellinor PT, et al. Utility of amino-terminal pro-brain natriuretic peptide, galectin-3, and apelin for the evaluation of patients with acute heart failure. J Am Coll Cardiol. 2006;48:1217-1224.

58. De Boer RA, Lok DJ, Jaarsma T, et al. Predictive value of plasma galectin-3 levels in heart failure with reduced and preserved ejection fraction. Ann Med. 2011;43:60-68.

59. Kindermann M, Reil JC, Pieske B, van Veldhuisen DJ, Böhm M. Heart failure with normal left ventricular ejection fraction: what is the evidence? Trends Cardiovasc Med. 2008;18:280-292.

60. Paulus WJ, Tschöpe C, Sanderson JE, et al. How to diagnose diastolic heart failure: a consensus statement on the diagnosis of heart failure with normal left ventricular ejection fraction by the Heart Failure and Echocardiography Associations of the European Society of Cardiology. Eur Heart J. 2007;28:2539-2550.

61. Ho JE, Liu C, Lyass A, et al. Galectin-3, a marker of cardiac fibro- 
sis, predicts incident heart failure in the community. J Am Coll Cardiol. 2012;60:1249-1256.

62. De Boer RA, Van Veldhuisen DJ, Gansevoort RT, et al. The fibrosis marker Galectin-3 and outcome in the general population. J Intern Med. 2012;272:55-64.

63. De Boer RA, Edelmann F, Cohen-Solal A, Mamas MA, Maisel A, Pieske B. Galectin-3 in heart failure with preserved ejection fraction. Eur J Heart Fail. 2013;15:1095-1101.

64. Vlassara H, Li YM, Imani F, et al. Identification of galectin-3 as a high-affinity binding protein for advanced glycation end products (AGE): a new member of the AGE-receptor complex. Mol Med. 1995;।: 634-646.

65. Hegab Z, Gibbons S, Neyses L, Mamas MA. Role of advanced glycation end products in cardiovascular disease. World J Cardiol. 2012;4:90-102.

66. Weigert J, Neumeier M, Wanninger J, et al. Serum galectin-3 is elevated in obesity and negatively correlates with glycosylated hemoglobin in type 2 diabetes. J Clin Endocrinol Metab.
2010;95:1404-1411.

67. Clementy N, Benhenda N, Piver E, et al. Serum galectin-3 levels predict recurrences after ablation of atrial fibrillation. Sci Rep. 2016; Sep 28; 6: 34357.

68. Lok DJ, Van Der Meer P, de la Porte PW, et al. Prognostic value of galectin-3, a novel marker of fibrosis, in patients with chronic heart failure: data from the DEAL-HF study. Clin Res Cardiol. 2010;99:323-328.

69. Gopal DM, Kommineni M, Ayalon N, et al. Relationship of plasma galectin-3 to renal function in patients with heart failure: effects of clinical status, pathophysiology of heart failure, and presence or absence of heart failure. J Am Heart Assoc. 2012;1:e000760.

70. Mann DL. Inflammatory mediators in heart failure: homogeneity through heterogeneity. Lancet. 1999;353:1812-1813.

71. Kang EH, Moon KC, Lee EY, et al. Renal expression of galectin-3 in systemic lupus erythematosus patients with nephritis. Lupus. 2009;18:22-28. 